\title{
Clonal relationships of CSF B cells in treatment-naive multiple sclerosis patients
}

\author{
Erica L. Eggers, Brady A. Michel, Hao Wu, Sheng-zhi Wang, Carolyn J. Bevan, Aya Abounasr, \\ Natalie S. Pierson, Antje Bischof, Max Kazer, Elizabeth Leitner, Ariele L. Greenfield, \\ Stanislas Demuth, Michael R. Wilson, Roland G. Henry, Bruce A.C. Cree, Stephen L. Hauser, and \\ H.-Christian von Büdingen \\ UCSF Weill Institute for Neurosciences, Department of Neurology, UCSF, San Francisco, California, USA.
}

\begin{abstract}
A role of B cells in multiple sclerosis (MS) is well established, but there is limited understanding of their involvement during active disease. Here, we examined cerebrospinal fluid (CSF) and peripheral blood (PB) B cells in treatment-naive patients with MS or high-risk clinically isolated syndrome. Using flow cytometry, we found increased CSF lymphocytes with a disproportionate increase of B cells compared with T cells in patients with gadolinium-enhancing $\left(\mathrm{Gd}^{+}\right)$lesions on brain MRI. Ig gene heavy chain variable region (Ig-VH) repertoire sequencing of CSF and PB B cells revealed clonal relationships between intrathecal and peripheral B cell populations, which could be consistent with migration of B cells to and activation in the CNS in active MS. In addition, we found evidence for bystander immigration of B cells from the periphery, which could be supported by a CXCL13 gradient between CSF and blood. Understanding what triggers B cells to migrate and home to the CNS may ultimately aid in the rational selection of therapeutic strategies to limit progression in MS.
\end{abstract}

Authorship note: E.L. Eggers and B.A. Michel contributed equally to this work.

Conflict of interest: During the study conception and execution, HCVB was full-time faculty at the UCSF. At the time of submission, $\mathrm{HCVB}$ was a full-time employee of $F$. Hoffmann-La Roche. HCVB has received compensation for consulting activities from Roche, Novartis, and Genzyme and research funding from Roche, Genentech, and Pfizer. SLH serves on the scientific advisory boards for Symbiotix, Annexon, Bionure, Neurona Therapeutics, and Molecular Stethoscope. SLH reports receiving travel reimbursement and writing assistance from F. HoffmannLa Roche for CD2O-related meetings and presentations. BACC has received compensation for consulting activities from Biogen, EMD Serono, and Teva.

Submitted: January 18, 2017

Accepted: October 17, 2017

Published: November 16, 2017

\section{Reference information:}

JCI Insight. 2017;2(22):e92724

https://doi.org/10.1172/jci.

insight. 92724

\section{Introduction}

The effectiveness of B cell-depleting antibodies as treatment in multiple sclerosis (MS) suggests that B cells play a central role in CNS myelin-directed autoimmunity (1-6). B cell repertoires in the periphery and CNS are closely linked in MS and other CNS autoimmune diseases, such as neuromyelitis optica, suggesting that disease-relevant B cell networks interact on both sides of the blood-brain barrier (7-11). Multiple features of intrathecal B cells, such as the presence of memory B cells, short-lived plasmablasts (12), and plasmablasts and plasma cells sharing the same B cell receptors (13), and the accumulation of somatic hypermutations (SHM) in Ig genes, support the participation of B cells in the CNS in antigen-directed immunity. Compared with peripheral blood (PB) or immune organs, B cell receptor diversity in cerebrospinal fluid (CSF) is generally limited, suggesting selective recruitment and/or survival of B cell populations in the MS CNS $(8,10,14)$.

Clonal IgG (oligoclonal bands [OCBs]) is present in the CSF of over 95\% of patients with MS. The presence and long-term persistence of OCBs indicate ongoing local production of $\operatorname{IgG}$ in the CNS, either by resident long-lived plasma cells or by antibody-secreting cells that continuously mature from an existing pool of memory B cells. Anti-CD20 therapy with rituximab reduces B cell numbers in CSF without eliminating OCBs (15); thus, intrathecal production of clonal IgG is largely a function of CD20-, long-lived plasma cells in CNS survival niches. Inflammatory infiltrates with lymphoid follicle-like structures are present in the meninges of patients with secondary progressive MS and are quite likely also present during the earlier relapsing phase of MS (16-18); such ectopic lymphoid tissues could support germinal center (GC) activity (19), plasma cell maturation, and survival. Interestingly, however, natalizumab, an anti-VLA4 antibody that limits lymphocyte transmigration across the blood-brain barrier and effectively decreases MS disease activity reduces intrathecal IgG production at least in some patients (20-22), which may suggest that OCB production also partially relies on peripheral stimuli.

Here, we studied the composition of the intrathecal and PB B cell compartments and performed Ig heavy chain variable region (Ig-VH) immune repertoire sequencing (Ig-RepSeq) to understand clonal relationships between B cell subsets on both sides of the blood-brain barrier in untreated MS patients. 
Table 1. Patient characteristics

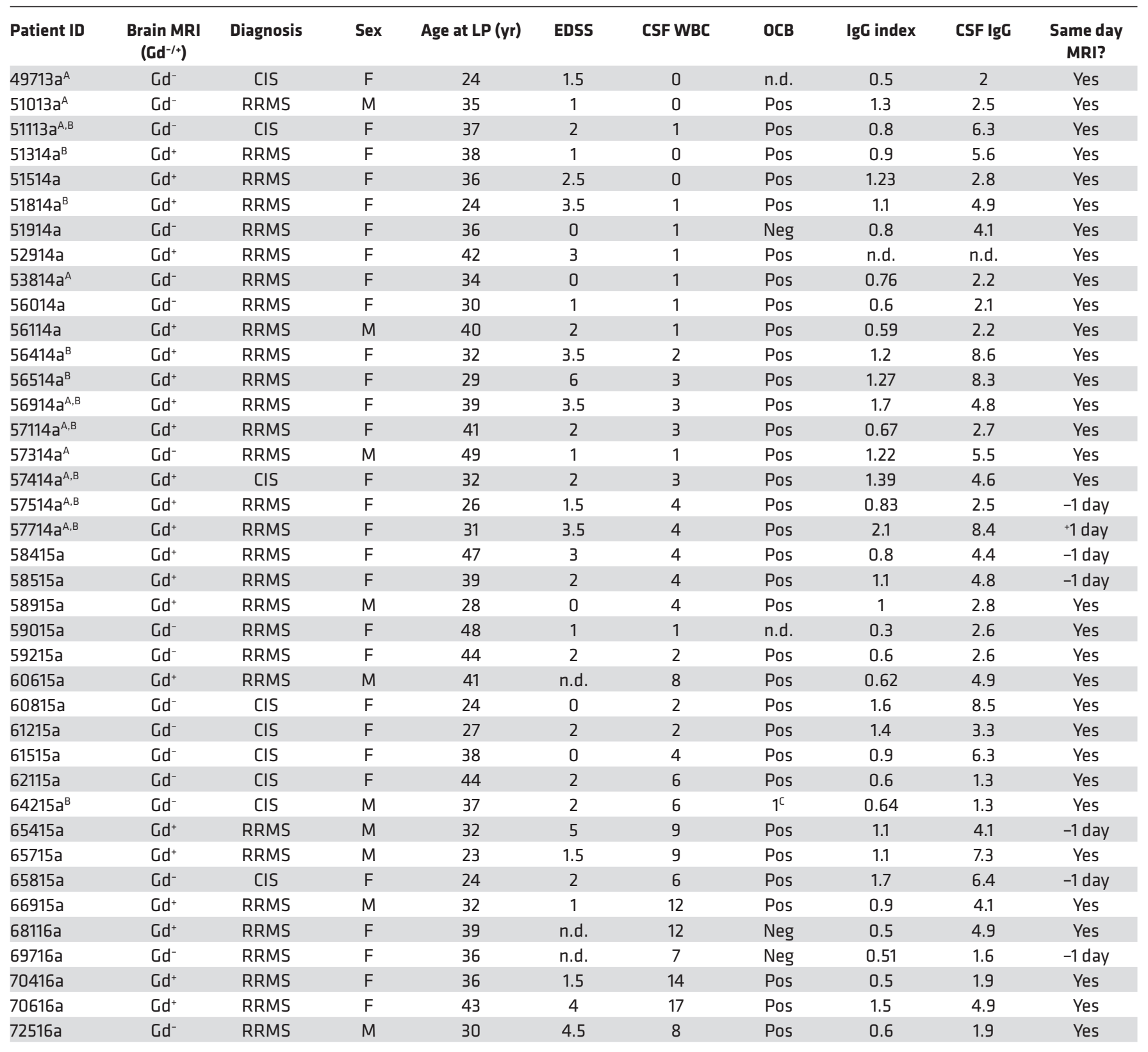

Shown are subject ID, diagnosis at time of study enrollment, sex, age, EDSS, OCB status, IgG index, CSF IgG levels (mg/dl), time point of MRI in relation to the LP, and whether the brain MRI showed Gd enhancement. Multiparameter flow cytometry was performed on each patient's PBMC and CSF lymphocytes. Footnotes refer to other experiments performed: ${ }^{A}$ cytokine/chemokine ELISA and ${ }^{B}$ Ig-RepSeq. ${ }^{C}$ One distinct band that was only present in CSF.

\section{Results}

Clinical findings. Characteristics of patients in our study are summarized in Table 1. The expanded disability status score (EDSS) was significantly higher in patients with Gd-enhancing $\left(\mathrm{Gd}^{+}\right)$lesions on brain MRI $(2.6 \pm 0.3 \mathrm{SD})$ compared with patients without $\mathrm{Gd}$ enhancement $\left(\mathrm{Gd}^{-}\right)(1.4 \pm 0.3 \mathrm{SD} ; P=0.009)$ (Table 2). Age, OCB, and IgG index were not different between $\mathrm{Gd}^{+}$and $\mathrm{Gd}^{-}$patient groups; CSFIgG levels were marginally higher in $\mathrm{Gd}^{+}$patients versus $\mathrm{Gd}^{-}$patients. Among $\mathrm{Gd}^{-}$patients, there were significantly more patients with a clinically isolated syndrome (CIS) versus a relapsing-remitting MS (RRMS) diagnosis (Table 2); however, virtually all patients with CIS in this study had OCB in their CSF, placing them in a high-risk category for clinically definite MS (23). 
Table 2. Comparison of patient characteristics between $\mathrm{Gd}^{+}$and $\mathrm{Gd}^{-}$patients

\begin{tabular}{lccc}
\hline & $\mathbf{G d}^{+}$ & $\mathbf{G d}^{-}$ & $\boldsymbol{P}$ \\
Age $(\mathrm{yr})$ & $35 \pm 6.5$ & $35.1 \pm 8$ & 0.96 \\
Sex, M/F & $6 / 16$ & $4 / 13$ & 1.0 \\
EDSS & $2.6 \pm 1.5$ & $1.4 \pm 1.2$ & 0.0095 \\
WBC & $5.4 \pm 4.8$ & $2.9 \pm 2.7$ & 0.065 \\
CSF-IgG & $4.7 \pm 2.0$ & $3.6 \pm 2.2$ & 0.093 \\
IgC index & $1.1 \pm 0.4$ & $0.9 \pm 0.4$ & 0.19 \\
OCB $/$ OCB- & $21 / 1$ & $13 / 1$ & 1.0 \\
CIS/RRMS & $1 / 21$ & $8 / 9$ & 0.005
\end{tabular}

Shown are mean values \pm SD or number of subjects meeting the indicated criteria. For linear measures and for group comparisons, a $t$ test and a Fisher's exact test were used to determine level of significance, respectively $(P)$.
$B$ cells are disproportionally increased in CSF compared with $T$ cells in active $M S$. We used multiparameter flow cytometry to characterize $\mathrm{B}$ and/or $\mathrm{T}$ lymphocytes in CSF. As previously shown (24), the percentage of B cells in CSF is significantly lower than that of T cells. Comparing $\mathrm{Gd}^{+}$and $\mathrm{Gd}^{-}$patients, we found significantly increased proportions of CSF B cells (Figure 1A) but not $\mathrm{T}$ cells (Figure 1B). In keeping with an increase of absolute numbers of CSF lymphocytes during an inflammatory state, numbers of $\mathrm{B}$ and $\mathrm{T}$ cells per $\mathrm{ml}$ were increased in $\mathrm{CSF}$ of $\mathrm{Gd}^{+}$patients versus $\mathrm{Gd}^{-}$patients (Figure 1, C and D). To understand whether $\mathrm{B}$ and $\mathrm{T}$ cells were equally increased in $\mathrm{Gd}^{+}$ CSF, we calculated the B/T cell ratio (Figure 1E) and found a disproportionate increase in $\mathrm{B}$ cell numbers in patients with $\mathrm{Gd}^{+}$MRI

$C D 27^{+} B$ cells dominate the CSF B cell compartment in MS. Overall, we found that CD19+ $\mathrm{B}$ cell subsets defined by CD27 and IgD expression differed substantially between CSF and PB irrespective of disease activity status (representative flow cytometry plots are shown in Figure 2, A and B), providing independent validation of previously published data (25). The proportion of $\mathrm{CD} 19^{+} \mathrm{B}$ cells among lymphocytes was significantly lower in CSF than in $\mathrm{PB}$ (Figure $2 \mathrm{C}$ ). The proportion of $\mathrm{CD}_{1}{ }^{+} \mathrm{CD} 27^{-} \mathrm{IgD}^{+}$naive $\mathrm{B}$ cells was lower in CSF (Figure 2D), whereas CD27 $7^{+} \mathrm{IgD}^{-}$Ig class-switched (SM) B cells and CD27 ${ }^{\text {hi }}$ plasmablasts/plasma cells (PC, CD27 $7^{\text {hi }}$ ) were significantly increased (Figure 2, G and H); the latter were mainly $\mathrm{CD} 38^{+}$and $\mathrm{CD} 138^{+}$(data not shown). $\mathrm{CD} 27^{+} \mathrm{IgD}^{+}$unswitched-memory (USM) and $\mathrm{CD} 27^{-} \mathrm{IgD}-$ "double-negative" (DN) B cells were slightly increased among CD19+ B cells in CSF (Figure 2, E and F).

Figure 1. CSF lymphocytes are dominated by T cells, but B cells are disproportionately increased in active MS. While most CSF lymphocytes are T cells, the overall proportion of B cells (CD19) (A) but not of T cells (CD3) (B) is significantly increased in $\mathrm{Gd}^{+}$versus $\mathrm{Gd}^{-}$patients. Reflective of an inflammatory state during active MS, the absolute numbers of both B cells (C) and T cells (D) are increased in $\mathrm{Gd}^{+}$patients. However, there is a disproportionate increase in B cells versus $T$ cells in active disease, as indicated by a significantly higher $\mathrm{B} / \mathrm{T}$ cell ratio, based on cell number per $\mathrm{ml}$ in $\mathrm{Gd}^{+}$patients $(\mathbf{E})$. Shown are data from patients with active MS (Gd+ lesions on brain MRI) and without Gd-enhancing (Gd-) lesions. Refer to Supplemental Table 1 for more information on the patients analyzed. Data are shown as scatter plots with mean $\pm 95 \% \mathrm{Cl}$. Comparisons were made using an unpaired $t$ test (GraphPad Prism); ${ }^{* *} P<0.01,{ }^{* * *} P<0.0001$.
A
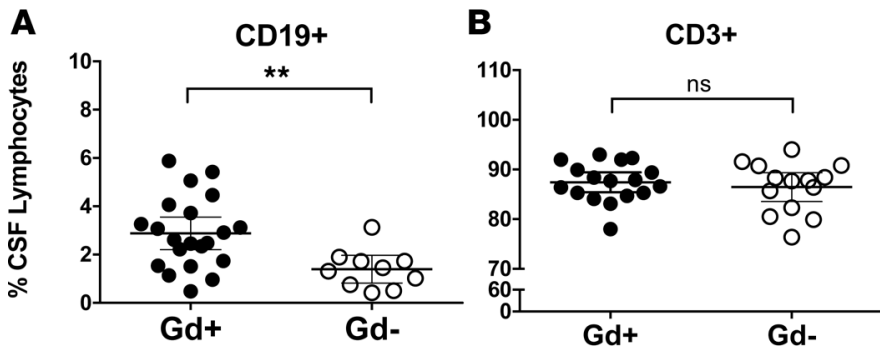

C
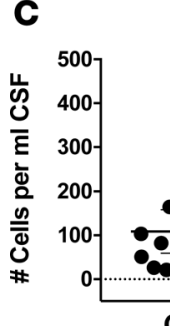

CD19+
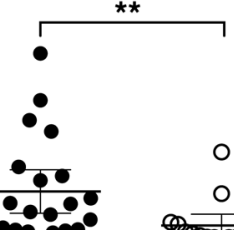

0

0

Gd+ Gd-

E

CSF $B / T$ cell Ratio

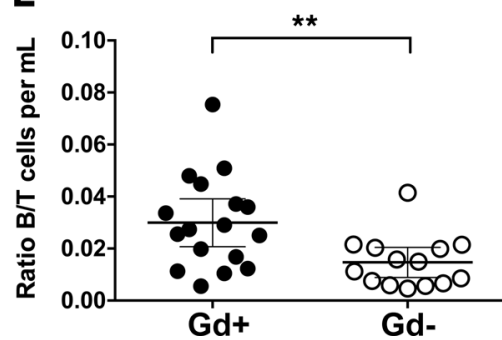

D

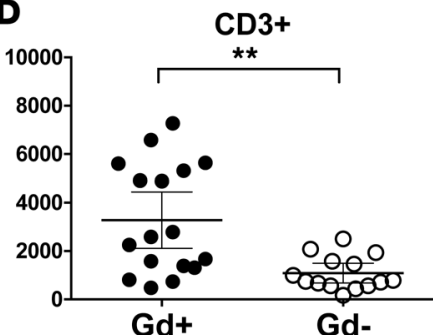


A

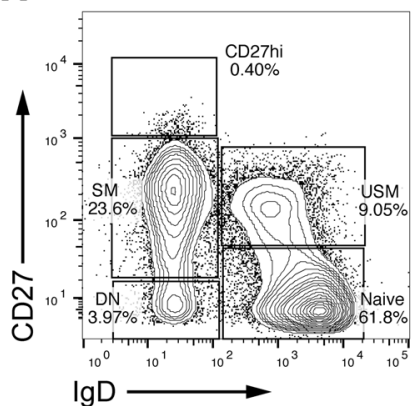

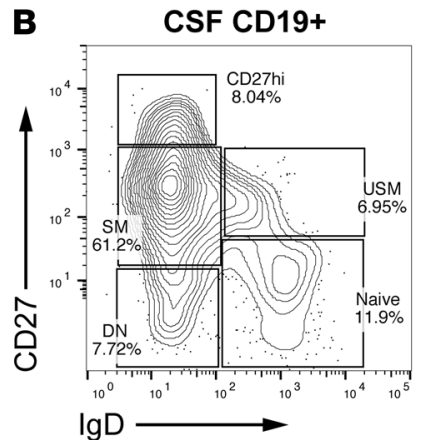

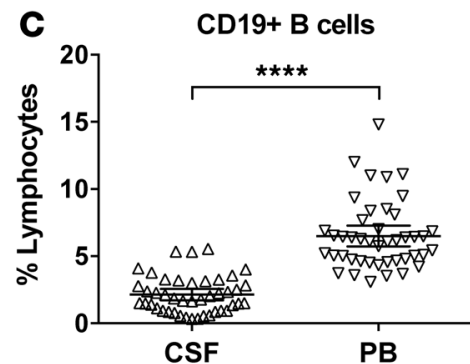

F $\quad$ CD27-lgD- (Double negative)

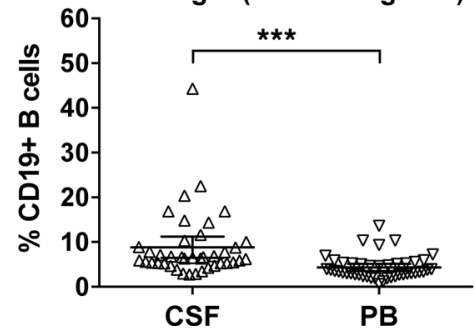

D

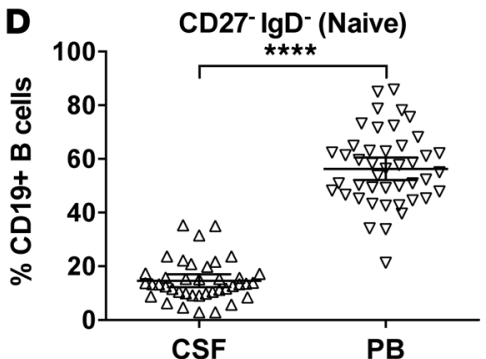

G $\mathrm{CD}^{+} 7^{+}$IgD- (Switched memory)

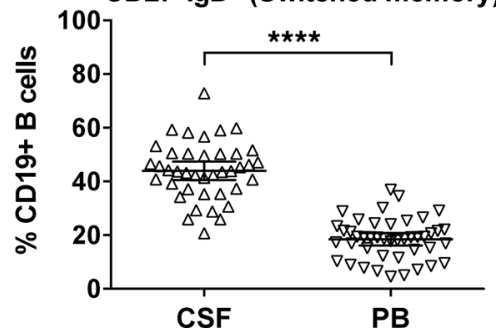

E $C D 27^{+} \lg \mathrm{D}^{+}$(Unswitched memory)
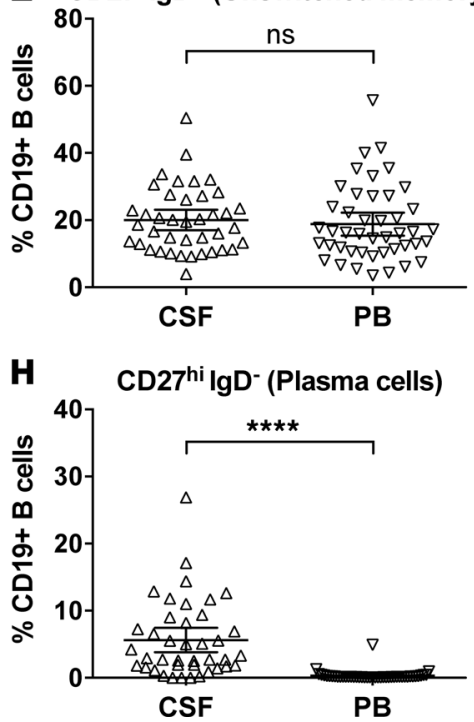

Figure 2. B cell subsets in CSF and PB. Shown are representative FACS plots of peripheral blood (PB) (A) and cerebrospinal fluid (CSF) (B) CD19+ B cell subsets (patient 56414), as defined based on the expression of IgD and CD27. The graphs in C-H summarize data from all patients, i.e., both Gd ${ }^{+}$and $\mathrm{Gd}$ patients combined, to provide an overall picture of B cell subset distribution in both compartments. CD19+ B cells are approximately 3-fold lower in CSF (C, $6.7 \% \pm 2.6 \%$ in PB versus $2.25 \% \pm 1.4 \%$ in CSF). The CSF contains B cell subpopulations predominantly that have undergone somatic rearrangement of their B cell receptors $(\mathbf{F}-\mathbf{H})$. Among $\mathrm{CD}{ }^{+} \mathrm{B}$ cells, in CSF compared with $\mathrm{PB}$, naive are approximately 5 -fold lower (D, 56.8\% $\pm 14.2 \%$ in PB versus $11.5 \% \pm$ $7.6 \%$ in CSF); USM are slightly increased (E, $19.1 \% \pm 11.7 \%$ in PB versus $22.8 \% \pm 10.4 \%$ in CSF); DN are increased $(\mathbf{F}, 4.0 \% \pm 2.5 \%$ in PB versus $7.7 \% \pm 7.3 \%$ in CSF); and SM are increased 2.5 -fold (G, $18 \% \pm 7.5 \%$ in PB versus $45.9 \% \pm 11.8 \%$ in CSF). Most significantly, the percentage of CD27 ${ }^{\text {hi }} B$ cells was increased $34-$ fold in CSF ( $\mathbf{H}, 0.2 \% \pm 0.2 \%$ in PB versus $7.6 \% \pm 7.9 \%$ in CSF). Refer to Supplemental Table 1 for more information on the patients analyzed. Data are shown as scatter plots with mean $\pm 95 \% \mathrm{Cl}$. Comparisons were made using paired $t$ tests; ${ }^{* *} P<0.01,{ }^{* * * *} P<0.0001$.

In accordance with a previous report by others (12), our findings demonstrate that the CSF B cell pool is composed primarily of memory and differentiated $\mathrm{B}$ cells capable of providing antigen-specific antigen-presenting cell-mediated (APC-mediated) and antibody-mediated immune functionality.

$P C s$ are increased in CSF of patients with $G d^{+} M R I$. Given the overall increased numbers of memory and $\mathrm{CD} 27^{\text {hi }} \mathrm{B}$ cells in MS CSF (Figure 2) and a disproportionate increase in B cells in $\mathrm{Gd}^{+}$CSF (Figure 1), we sought to determine whether there is a balanced increase in all $\mathrm{B}$ cell subsets during active MS evidenced by a Gd ${ }^{+}$MRI. The absolute number of cells per ml was only significantly increased in SM and CD2 $7^{\text {hi }}$ subsets (Figure 3, D and E) , and not significantly higher in naive, USM or double negative subsets (Figure 3, A-C). When further examining the averages for each subset in $\mathrm{Gd}^{+} \mathrm{CSF}$ versus $\mathrm{Gd}^{-} \mathrm{CSF}$, we found a greater, approximately 8-fold, relative increase of $\mathrm{CD} 27^{\text {hi }} \mathrm{B}$ cells compared with an approximately 3-fold increase of SM B cells (Figure 3F). In PB, we found B cell subsets more similarly distributed between $\mathrm{Gd}^{+}$and $\mathrm{Gd}^{-}$ patients, with significant differences only in CD27- $\mathrm{IgD}^{-} \mathrm{DN}$ B cells (Supplemental Figure 1; supplemental material available online with this article; https://doi.org/10.1172/jci.insight.92724DS1).

Clonal relationships between CSF and PB suggest influx of functionally diverse B cell populations. The finding that increased CSF B cells were linked to the presence of $\mathrm{Gd}^{+}$lesions on brain MRI suggested their association with disease activity in MS and raised the question whether there are clonal 
A
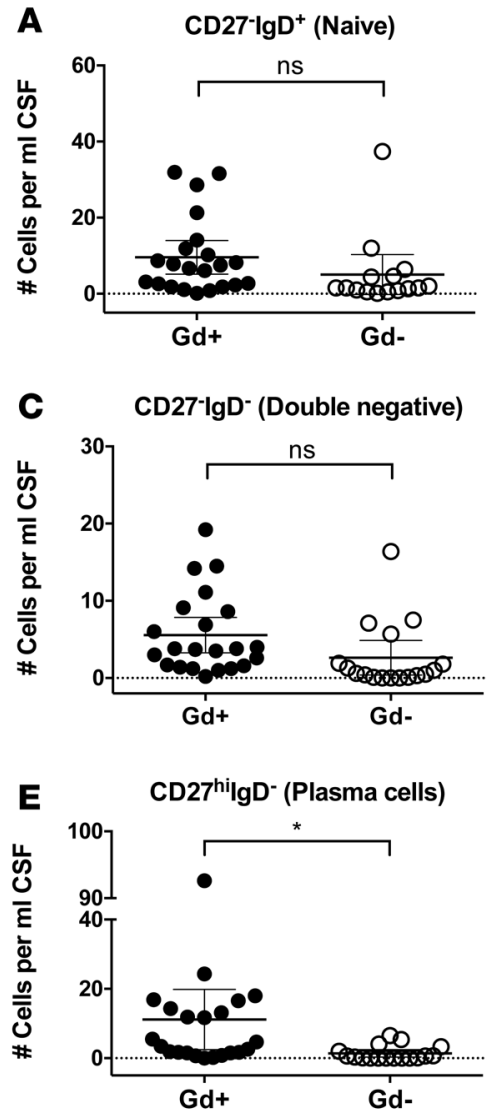

B

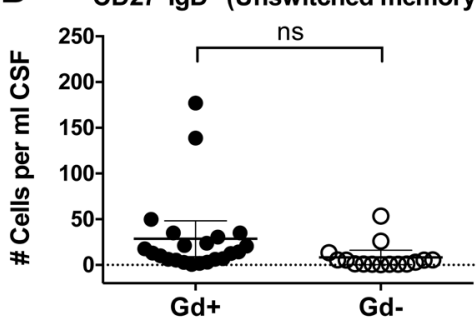

D

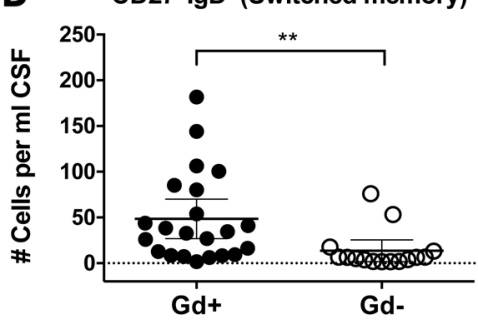

$\mathbf{F}$

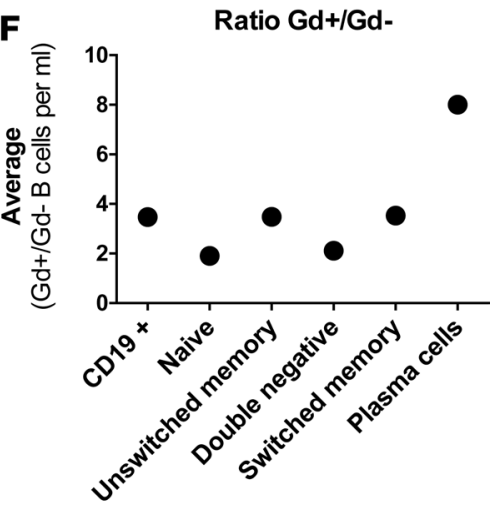

Figure 3. The abundance of antigen-induced $B$ cells is increased in Gd+ CSF. In CSF of patients with Gd-enhancing lesions on brain MRI, B cell subsets resulting from antigen-driven stimulation are increased in numbers ( $\mathbf{D}$ and $\mathbf{E}$ ). Other $B$ cell subpopulations appeared increased as well but not in a significant fashion (A-C). (F) The fold difference of the mean number of each $B$ cell subset between $\mathrm{Gd}^{+}$and $\mathrm{Gd}^{-} \mathrm{CSF}$, showing the greatest increase of CD27 hi B cells. Shown are scatter plots with each point representing findings from a single patient, and all data are shown as mean $\pm 95 \% \mathrm{Cl}$, except for those in $\mathbf{E}$, where the ratio of means is shown and therefore no error bars can be calculated. Refer to Supplemental Table 1 for more information on the patients analyzed in A-E. Comparisons were made using an unpaired $t$ test; ${ }^{*} P<0.05,{ }^{* *} P<0.01$.

relationships between CSF B cell subsets and their PB counterparts. To address this question, we performed Ig-RepSeq on CSF and PB samples (Table 1). PB subsets (naive, USM, DN, SM, CD27 hi) were sequenced from 11 patients. Matched bulk-sorted CSF B cells (i.e., all subsets sorted together in the same tube) were sequenced from 3 of these 11 patients (51113a, 51314a, and 51814a), CSF B cells sorted as 4 subsets (naive, USM, DN, SM/CD2 $7^{\text {hi }}$ combined) from 2 of 11 patients (56414a and 56514a), and CSF B cell subsets sorted as 5 separate subsets (naive, USM, DN, SM, CD27 ${ }^{\text {hi }}$ ) from 6 of 11 patients (56914a, 57114a, 57414a, 57514a, 57714a, and 64215a). Using IGHV gene-specific forward primers and IgM or IgG constant region reverse primers (Table 3), extensive IgM/G-VH cDNA libraries were generated and then sequenced using an IonTorrent PGM sequencer (Supplemental Table 2). In total, we generated and analyzed nearly $1.3 \times 10^{7} \mathrm{Ig}$-VH sequences. In general, next-generation sequencing technologies are error-prone (26), which, in the case of Ig-VH repertoire sequencing, can lead to overestimation of SHM and Ig repertoire diversity. We applied our bioinformatics pipeline to first discard nonproductive (i.e., incorrect reading frame) sequencing reads. In a next step, we determined IGHV/IGHJ usage, H-CDR3 amino acid sequence, and the number of mutations away from the closest IGHV germline segment (SHM) for each sequence; this information, along with the B cell subset origin (i.e., naive, SM, USM, DN, CD27 $7^{\text {hi }}$ ) and isotype (i.e., IgG, $\operatorname{IgM}$ ), was used to annotate each productive Ig-VH read. SHM profiles generated for each PB subset largely replicated what would be expected biologically (Figure 4), i.e., low SHM counts in naive B cell IgM-VH and increasing SHM in more mature B cells, with the highest number of SHMs being present in IgG-expressing CD2 $7^{\text {hi }} B$ cells (Figure 4A). USM B cells had also accumulated some level of SHM, despite not having undergone class-switch recombination (Figure 4B). SHM profiles of CSF B cell subsets were reflective of the limited diversity found in this compartment (Figure 4, C and D). 
Table 3. Primer sequences used for Ig-RepSeq library generation

\begin{tabular}{|c|c|}
\hline \multicolumn{2}{|r|}{ Forward primers } \\
\hline General design & 5'-trP1 adaptor-ICHV1-7-3' \\
\hline VH1 & 5'-CCTCTCTATGGGCAGTCGGTGAT-GAARRTYTCCTCCAAGGYWTC-3' \\
\hline VH3 & 5'-CCTCTCTATGGGCAGTCGGTCAT-KARACTCTCCTCTRCAGCCTB-3' \\
\hline VH4 & 5'-CCTCTCTATGGGCAGTCGGTGAT-GTCCCTCACCTGCRCTGTCTM-3' \\
\hline VH5 & 5'-CCTCTCTATGGGCAGTCGGTCAT-GARGATCTCCTCTAAGGGTTC-3' \\
\hline \multicolumn{2}{|r|}{ Reverse primers } \\
\hline General design & 5'-adaptor-barcode+GAT key-lgG/M gene-specific sequence-3' \\
\hline IgG & 5'-CCATCTCATCCCTGCGTCTCTCCCACTCAG-barcode-CAT-GGGAAGACSGATGGGCCCTTCGTCG-3' \\
\hline $\lg M$ & 5'-CCATCTCATCCCTCCGTGTCTCCGACTCAG-barcode-GAT-GGAGTCGGGAAGGAAGTCCTGTGCGAG-3' \\
\hline
\end{tabular}

To identify clonal overlap between CSF and PB B cells based on Ig-RepSeq data, we used a previously published distance-metric clustering approach (Hamming distance; see Methods), placing all Ig-VH with related H-CDR3 and identical IGHV and IGHJ usage in clusters of clonally related Ig-VH (Ig-VH clusters). This approach is insensitive to possible sequencing errors in Ig-VH reads, as clonally related Ig-VH are combined in their appropriate cluster irrespective of whether SHM or sequencing errors altered the Ig-VH. Clonal relationships were identified between different CSF and PB B cell subsets in each patient. Figure 5 shows overviews of clonal relationships between CSF and PB B cell subsets for all patients from which Ig-RepSeq data was available (Figure 5A). Supplemental Table 3 lists the frequencies of clonal connections among each B cell subpopulation shown in Figure 4. Supplemental Table 4 lists the characteristics of CSF Ig-VH clusters, whether they were exclusively found in CSF, or to which PB B cell subsets clonal relationships were identified. IGHV usage distribution per analyzed patient and sample/subset is shown in Supplemental Figure 2.

Increased CSF B cells were correlated with overall number of CSF Ig-VH clusters $\left(\mathrm{R}^{2}=0.633, P=\right.$ 0.0034) (Figure 5B) and with CSF "restricted" Ig-VH clusters (i.e., clusters for which no related sequences were found in the matched $\mathrm{PB}$ sample) $\left(\mathrm{R}^{2}=0.6675, P=0.021\right)$ (Figure $\left.5 \mathrm{C}\right)$ and partially correlated with the proportion of PB Ig-VH clusters with clonal relationships to the CSF $\left(\mathrm{R}^{2}=0.4044, P=0.0355\right)$ (Figure 5D). These findings suggest that influx and intrathecal expansion of B cells occur during active MS.

We found clonally related Ig-VH expressed by CSF CD27 $7^{\text {hi }}$ and peripheral B cell subsets in all patients from which $\mathrm{CD} 27^{\text {hi }} \mathrm{B}$ cells could be sorted (all were $\mathrm{Gd}^{+}$) (Figure 6), suggesting that peripherally antigen-stimulated B cells may have migrated to the CSF compartment where they became activated to further mature into antibody-secreting $\mathrm{CD} 27^{\text {hi }} \mathrm{B}$ cells. In addition, we identified clusters of related Ig-VH that were exclusively expressed by CSF B cells, including some found in CSF SM and CD27 ${ }^{\text {hi }}$ B cells, which may point toward intrathecal activation without a peripheral contribution (Figure 6). In $6 \mathrm{Gd}^{+}$patients, we identified Ig-VH clusters containing IgM-VH and IgG-VH derived from CSF B cells, possibly indicating intrathecal Ig class-switch recombination (Supplemental Table 3).

Interestingly, we also found substantial clonal overlap between CSF USM B cells and PB USM, SM, and DN B cells but only a limited clonal relatedness of CSF USM to CSF CD27 ${ }^{\text {hi }}$ or SM B cells (Figure 6); SHM profiles of USM B cells were most closely related to SHM profiles of IgM-expressing PC and SM B cells (Figure 4B). We were able to sort naive B cells from CSF and obtain IgM-VH sequences from 4 patients; as was the case for USM B cells, clusters containing CSF naive B cells were almost exclusively clonally connected to the periphery and did not include post-GC CSF B cells, potentially suggesting bystander influx without further intrathecal stimulation (Figure 6 and Supplemental Figure 3). The presence of related Ig-VH expressed by naive B cells in CSF and by peripheral B cells also supports homeostatic proliferation (i.e., nonantigen-driven clonal expansion) of naive $\mathrm{B}$ cells.

$B$ cell CXCR5 and CSF CXCL13 as possible drivers of B cell migration to the CNS during active MS. Finally, we explored the possibility that CXCR5 on B cells and CXCL13 in CSF might be involved in B cell recruitment to CSF during active MS by measuring CXCL13 levels in CSF obtained usually on the same day of the brain MRI. In alignment with the finding that increased B cell migration to the CSF occurs in active MS, we 


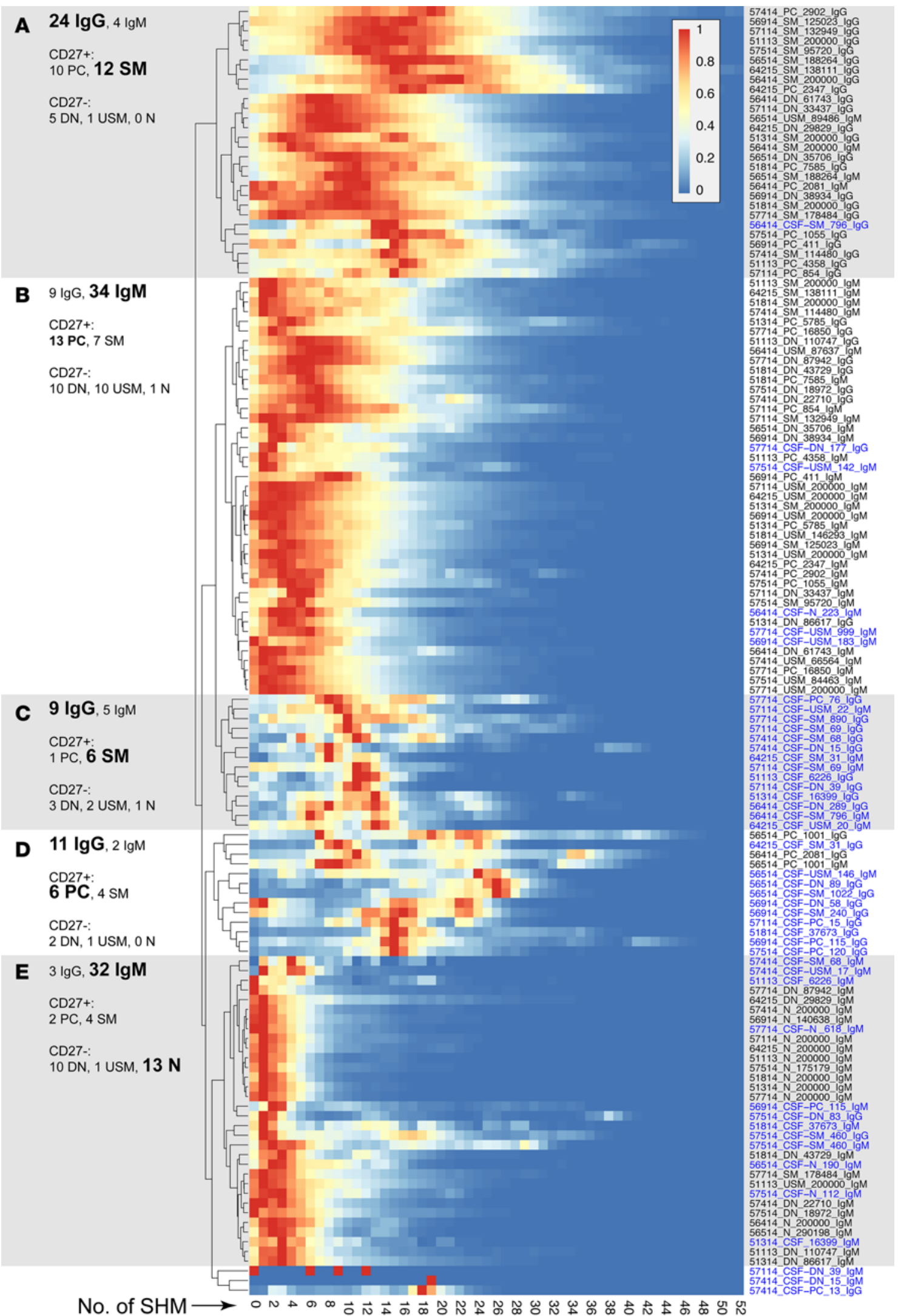

Figure 4. Hierarchical clustering of SHM profiles. Profiles of SHM along the analyzed IGHV portion of Ig-VH were normalized to the peak (=1, red) and subjected to unsupervised hierarchical clustering. In general, post-GC subsets display higher and naive B cells display lower levels of SHM. (A) Higher level SHM can be found in IgG-expressing SM B cells and PCs. (B) Lower level SHM can be found in IgM-expressing SM B cells and PCs. (C and D) Predominantly IgG-expressing CSF-derived SM B cells and PCs form separate clusters. (E) Lowest level SHM can be found among mostly IgM-expressing naive B cells. 
Each row represents an individual B cell sample, as indicated by sample names containing patient ID, B cell subset, number of cells analyzed, and Ig isotype separated by “_." CSF B cells subsets are indicated by "CSF" before the subset designation and blue type. N, naive B cells; USM, unswitched-memory B cells; DN, double-negative B cells; SM, switched-memory B cells; PC, plasma cells/plasmablasts.

found levels of CXCL13 in CSF of $\mathrm{Gd}^{+}$patients $(n=5)$ that were similar to plasma CXCL13 levels, while no CXCL13 was detectable in $\mathrm{Gd}^{-} \mathrm{CSF}(n=5)$ using a commercial ELISA assay (Figure 7A). The receptor for CXCL13, i.e., CXCR5, was identified on most B cells (Figure 7B); however, significantly fewer CSF and PB CD27 ${ }^{\text {hi }}$ B cells expressed CXCR5. To assess whether the presence of CSF CXCL13 in $\mathrm{Gd}^{+}$CSF was merely due to increased passive exchange of soluble factors from the periphery across a compromised blood-brain barrier, we also measured other chemokines and cytokines of similar or higher molecular weight as CXCL13 $(10.7 \mathrm{kDa})$, i.e., CXCL12 (8.5 kDa), BAFF (31.2 kDa), and APRIL (27.4 kDa) (Figure 7, C-E). We found that CXCL12 and BAFF levels were lower in CSF than PB independent of Gd enhancement, while APRIL may even be slightly, though not statistically significantly, increased in $\mathrm{Gd}^{-} \mathrm{CSF}$. Overall, these data suggest that CXCL13 in $\mathrm{Gd}^{+}$CSF is produced locally in the CSF or CNS compartments and may be involved in the recruitment of diverse populations of CXCR5-expressing B cells during active MS, except for CD27 ${ }^{\text {hi }} \mathrm{B}$ cells.

\section{Discussion}

We performed comprehensive analyses of CSF and peripheral B cells in patients with a new diagnosis of RRMS or high-risk CIS who had never been treated with disease-modifying therapy for MS. Most patients (34 of 39) had OCBs in their CSF, suggesting a preestablished intrathecal plasma cell repertoire as potential evidence for immune activation and CNS infiltration that may have occurred prior to CSF sampling.

With regards to the CSF lymphocyte composition, we confirmed that $\mathrm{T}$ lymphocytes are much more prevalent than B cells in CSF of MS patients, that absolute numbers of both B and T cells are increased in active MS (27), and that the CSF is enriched with memory B cells (28). In patients with $\mathrm{Gd}^{+}$lesions on brain MRI, we found a disproportionate increase in B cell numbers compared with $\mathrm{T}$ cells, suggesting that $\mathrm{B}$ cell immigration and/or activation is associated with active MS. We found increased CD27 ${ }^{\text {hi }} \mathrm{B}$ cells in CSF, confirming earlier reports that intrathecal plasma cells are associated with active CNS inflammation in MS $(12,29)$ and suggesting antigen-driven B cell activation. This is interesting considering that plasma cells are antibody-secreting cells and that the pathological involvement of antibodies in MS remains poorly understood (30). Antibody-secreting cells are clonally expanded in the CSF where they participate in the production of clonal IgG (i.e., OCBs) $(7,31,32$ ); their presence in the CSFs studied here and their apparent association with MS disease activity further support antigen-driven and possibly antibody-mediated mechanisms as playing a key role in MS immune pathology.

We previously showed that clonally related B cells are present in CSF and PB of MS patients (7, 8, 10). Here, we were interested in the clonal relationships between B cell subsets in untreated MS CSF and PB. In B cells, random somatic recombination of Ig germline genes (IGHV, IGHD, IGHJ), SHM, and Ig class-switch recombination shape the highly diverse B cell repertoire and generate readily identifiable and specific molecular signatures that can be exploited for the identification of clonally related B cells in spatially separated compartments and/or different B cell subsets. We applied flow cytometry in combination with Ig-RepSeq to identify clonally related B cells in CSF or PB. Our results suggest that during episodes of disease activity, diverse populations migrate to and/or are stimulated within the CNS/CSF compartment.

Unresolved questions are how MS relapses are triggered and whether antigen-specific or nonspecific restimulation is required for relapsing disease activity. Our finding of a preexisting network of potentially disease-driving immune cells on both sides of the blood-brain barrier could explain how relapsing immune activity is facilitated, such as when patients experience relapses during systemic infection $(33,34)$. Indeed, we found clonally related CD27 $7^{\text {hi }}$ PCs in PB and CSF that were also related to Ig class-switched-memory B cells in the same compartments. Given that CD27 $7^{\text {hi }}$ PCs are likely restricted to their compartment (as evidenced by their low CXCR5 expression), and the persistence of memory B cells in MS CSF and CNS in general, parallel maturation of preexisting SM B cells on both sides of the blood-brain barrier may have occurred following a peripheral immune activating event. The presence of clonal relationships between memory B cells and CD27 $7^{\text {hi }}$ plasma cells in the CSF and CSF Ig-VH clusters containing related IgM-VH and IgG-VH also support local B cell activation and subsequent maturation and class-switch recombination.

Clearly, the MS disease process is complex, with multiple mechanisms contributing to relapsing activity. The efficacy of natalizumab in suppressing MS relapses supports common thinking that peripheral activation, transmigration, and intrathecal maturation of SM B cells to CD27 $7^{\text {hi }}$ PCs also contributes to MS 
A
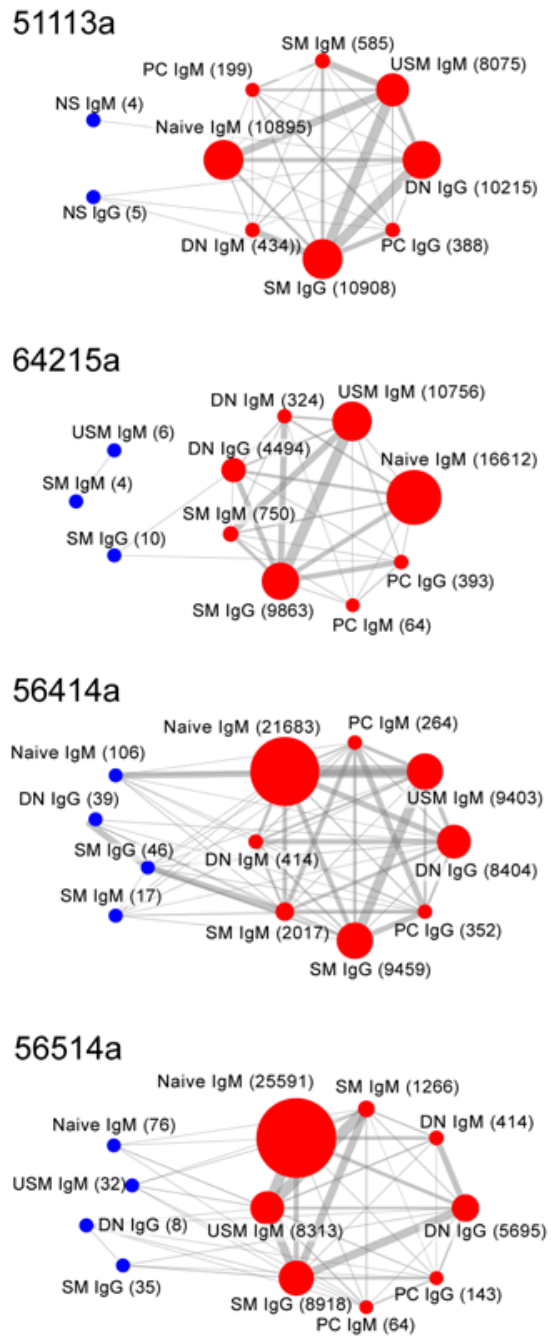

B

Total CSF clusters

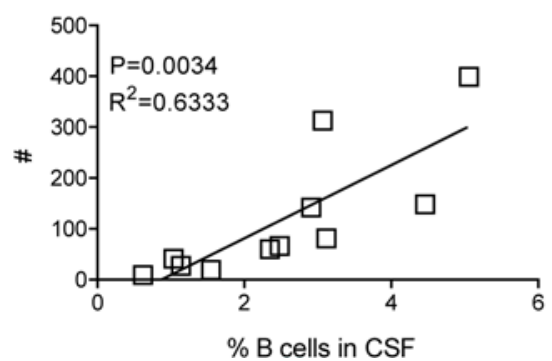

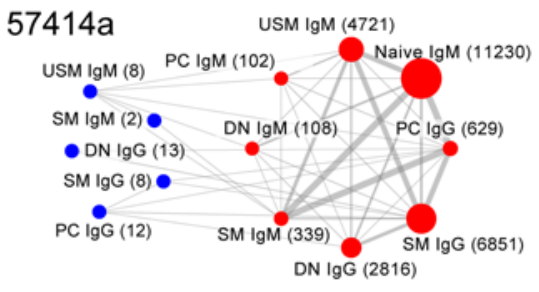

$51814 a$

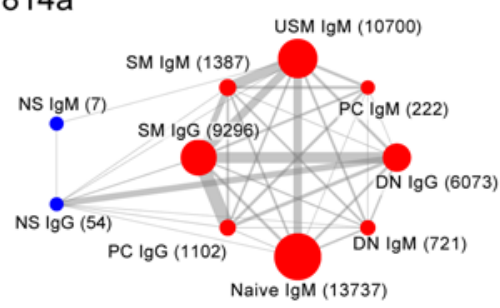

$57514 a$
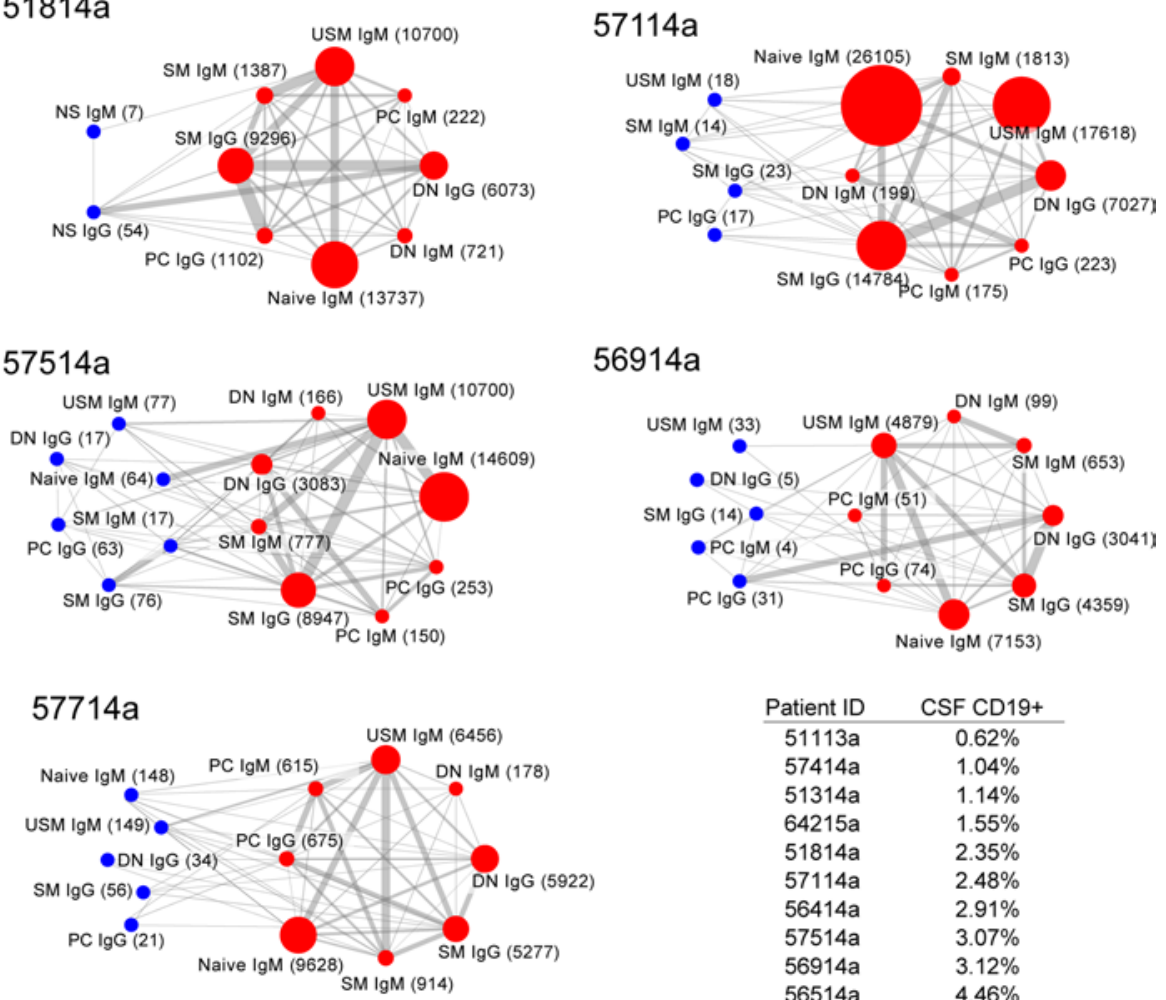

\begin{tabular}{cc} 
Patient ID & CSF CD19+ \\
\hline $51113 a$ & $0.62 \%$ \\
$57414 a$ & $1.04 \%$ \\
$51314 a$ & $1.14 \%$ \\
$64215 a$ & $1.55 \%$ \\
$51814 a$ & $2.35 \%$ \\
$57114 a$ & $2.48 \%$ \\
$56414 a$ & $2.91 \%$ \\
$57514 a$ & $3.07 \%$ \\
$56914 a$ & $3.12 \%$ \\
$56514 a$ & $4.46 \%$ \\
$57714 a$ & $5.06 \%$
\end{tabular}

\section{C $\quad$ CSF restricted clusters}

\section{PB clusters connecting to CSF}

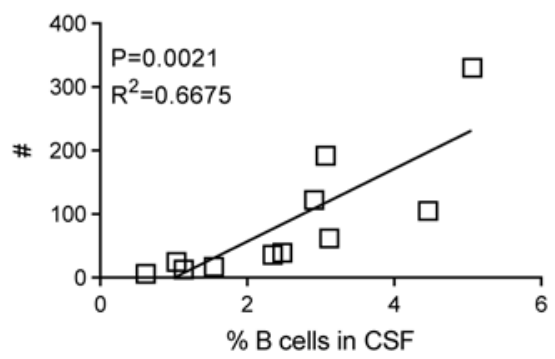

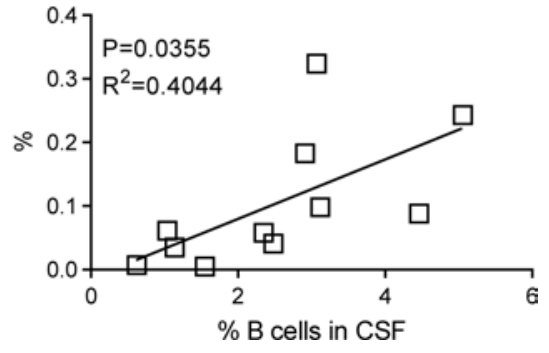

Figure 5. Increased CSF B cells correlate with CSF B cell diversity and B cell influx. (A) From the top left to bottom right, networks are ordered per percentage of CD19+ B cells among CSF lymphocytes. Nodes represent CSF (blue) or PB (red) B cell subsets subjected to Ig-RepSeq (NS, nonsorted; N, naive B cells; DN CD27-lgD- double-negative B cells; SM, switched-memory B cells; CD27hi, plasmablasts/plasma cells); for each population, relevant Ig isotypes were obtained by using IgG- and IgM-specific primers. Numbers in parentheses represent the number of Ig-VH clusters identified per subpopulation. Edges (lines) between nodes indicate clonal connections between subsets. Node size and edge thickness are relative to the number of Ig-VH clusters or connections, respectively. The displayed networks greatly simplify the highly complex picture of clonal relationships between B cell subsets; see Supplemental Table 3 for a listing of all CSF clusters and respective clonal connections to CSF and/or PB B cell subsets. (B) Correlation between CSF B cell proportion and total number of CSF B cell clusters. (C) Correlation between CSF B cell proportion and number of B cell clusters that can be exclusively found in the CSF. (D) Correlation between CSF B cells and proportion of peripheral blood B cells that form a connection to the CSF compartment (B cell influx). Standard linear regression was used to determine goodness of fit and significance. 


\begin{tabular}{|c|c|c|c|c|c|c|c|}
\hline & \multicolumn{2}{|l|}{ Naive } & \multicolumn{2}{|l|}{ USM } & \multicolumn{3}{|c|}{ CD27hi } \\
\hline $56414 a$ & Naive IgM & $\begin{array}{l}\text { Naive IgM } \\
\text { USM IgM } \\
\text { DN IgM } \\
\text { DN IgG } \\
\text { SM IgM } \\
\text { SM IgG } \\
\text { CD27hi IgM } \\
\text { CD27hi lgG }\end{array}$ & n.a. & & & n.a. & \\
\hline $56514 a$ & Naive $\lg \mathrm{M}$ & $\begin{array}{l}\text { Naive IgM } \\
\text { USM IgM } \\
\text { SM IgM } \\
\text { CD27hi IgM } \\
\text { CD27hi IgG }\end{array}$ & USM IgM & $\begin{array}{l}\text { Naive IgM } \\
\text { USM IgM } \\
\text { SM IgM } \\
\text { SM IgG } \\
\text { CD27hi IgM }\end{array}$ & & n.a. & \\
\hline $56914 a$ & n.a. & & 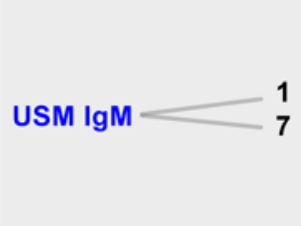 & $\begin{array}{l}\text { Naive IgM } \\
\text { USM IgM }\end{array}$ & $\begin{array}{l}\text { SM IgG } \\
5 \mid 1 \\
\text { CD27hi IgM/G }\end{array}$ & $\begin{array}{l}2 \\
3 \\
-7 \\
-11 \\
1 \\
2\end{array}$ & $\begin{array}{l}\text { Naive IgM } \\
\text { USM IgM } \\
\text { DN IgG } \\
\text { SM IgG } \\
\text { CD27hi IgM } \\
\text { CD27hi IgG }\end{array}$ \\
\hline 57114a & n.a. & & $\begin{array}{l}\text { USM IgM } \\
\left.\quad 2\right|^{\text {SM IgM }}\end{array}$ & $\begin{array}{l}\text { Naive IgM } \\
\text { USM IgM } \\
\text { DN IgG } \\
\text { SM IgM } \\
\text { SM IgG } \\
\text { CD27hi IgM }\end{array}$ & $\begin{array}{l}\text { SM IgG } \\
2 \mid 0 \\
\text { CD27hi IgG }\end{array}$ & $\begin{array}{r}3 \\
8 \\
-1 \\
5\end{array}$ & $\begin{array}{l}\text { DN IgG } \\
\text { SM IgG } \\
\text { CD27hi IgM } \\
\text { CD27hi IgG }\end{array}$ \\
\hline $57414 a$ & n.a. & & $\begin{array}{l}\text { USM IgM } \\
1 \\
\text { SM IgM }\end{array}$ & $\begin{array}{l}\text { USM IgM } \\
\text { DN IgM } \\
\text { SM IgM } \\
\text { CD27hi IgM } \\
\text { CD27hi IgG }\end{array}$ & $\begin{array}{l}\text { SM IgG } \\
1 \mid 1 \\
\text { CD27hi IgG }\end{array}$ & $\begin{array}{r}1 \\
-4 \\
2\end{array}$ & $\begin{array}{l}\text { DN IgG } \\
\text { SM IgG } \\
\text { CD27hi IgG }\end{array}$ \\
\hline $57514 a$ & Naive IgM & $\begin{array}{l}\text { Naive IgM } \\
\text { USM IgM } \\
\text { CD27hi IgM } \\
\text { CD27hi IgG }\end{array}$ & $\begin{array}{l}\text { USM IgM } \\
10 \\
\text { SM IgM }\end{array}$ & $\begin{array}{l}\text { Naive IgM } \\
\text { USM IgM } \\
\text { DN IgG } \\
\text { SM IgM } \\
\text { SM IgG } \\
\text { CD27hi IgM }\end{array}$ & $\begin{array}{c}\text { DN IgG } \\
5 \mid 3 \\
\text { CD27hi IgG } \\
7 \mid 1 \\
\text { SM IgM/G }\end{array}$ & $\begin{array}{l}2 \\
3 \\
2 \\
16 \\
16 \\
2 \\
26 \\
4 \\
14\end{array}$ & $\begin{array}{l}\text { Naive IgM } \\
\text { USM IgM } \\
\text { DN IgM } \\
\text { DN IgG } \\
\text { SM IgM } \\
\text { SM IgG } \\
\text { CD27hi IgM } \\
\text { CD27hi IgG }\end{array}$ \\
\hline $57714 a$ & $\begin{array}{cr}\text { Naive IgM } & 2 \\
2 & 2 \\
\text { USM IgM } & 2 \\
& 2\end{array}$ & $\begin{array}{l}\text { Naive IgM } \\
\text { USM IgM } \\
\text { DN IgG } \\
\text { SM IgM } \\
\text { SM IgG } \\
\text { CD27hi IgM } \\
\text { CD27hi IgG }\end{array}$ & $\begin{array}{l}\text { Naive IgM } \\
\quad 2 \\
\text { USM IgM }\end{array}$ & $\begin{array}{l}\text { Naive IgM } \\
\text { USM IgM } \\
\text { CD27hi IgM } \\
\text { DN IgG } \\
\text { SM IgM } \\
\text { SM IgG } \\
\text { CD27hi IgG }\end{array}$ & $\begin{array}{l}\text { SM IgG } \\
7 \mid 4 \\
\text { CD27hi IgG }\end{array}$ & $\begin{array}{r}3 \\
-1 \\
-4\end{array}$ & $\begin{array}{l}\text { SM IgG } \\
\text { CD27hi IgM } \\
\text { CD27hi IgG }\end{array}$ \\
\hline
\end{tabular}

Figure 6. Clonal relationships between CSF naive, USM, and CD27 ${ }^{\text {hi }}$ B cells and other CSF or peripheral blood B cells suggest recruitment of antigen-specific and nonspecific B cells. CSF B cell subsets are in blue type; PB subsets are in red type. Numbers represent number of clusters identified containing clonally related Ig-VH from both connected subsets; in the CD27 $7^{\text {hi }}$ column, blue numbers represent CSF-restricted Ig-VH clusters, red numbers represent clusters comprising CSF and PB subsets. For simplicity, only the direct connections between the CSF subset of interest (bold blue type) and other subsets are shown. See Supplemental Figure 3 for an example detailed overview of clonal connections between CSF-naive B cells and other 
CSF or PB subsets (patient 56414). For comprehensive information on clonal relationships between CSF B cells and other subsets see Supplemental Table 4. n.a., not available, i.e., CSF subset not obtained flow cytometry sorting; N, naive B cells; USM, unswitched-memory B cells; DN, double-negative B cells; SM, switched-memory B cells; PC, plasma cells/plasmablasts.

disease activity (35). Our finding of a positive correlation between bicompartmental B cell clusters and the proportion of CSF CD19+ B cells suggests that peripherally activated B cells may be directly contributing to intrathecal inflammation and B cell activation in MS. Together with previous evidence of intrathecal IgMand IgG-VH SHM (10,36-38), our findings add to a model (Figure 8) of B cell activation that may originate in the periphery, continue in the CNS compartment, partially involve preexisting intrathecal memory $\mathrm{B}$ cells, and may also attract antigen nonspecific subsets to the CNS compartment.

Interestingly, while we did find naive CSF B cells with clonally related counterparts in the periphery, naive $B$ cells do not seem to become stimulated intrathecally to mature to memory B cells. Thus, GC functionality may only be partially represented intrathecally, providing support for class-switch recombination, SHM (19), and plasma cell maturation but not for further differentiation or survival of naive B cells. The latter point is also supported by the fact that, contrary to $\mathrm{PB}$, naive $\mathrm{B}$ cells are among the least abundant $\mathrm{B}$ cells in CSF. Ig non-class-switched IgD-expressing memory B cells (USM) have been described in MS CSF (25). Here, we found that CSF USM B cells are clonally related to a variety of peripheral B cell subsets, including IgG-expressing $\mathrm{SM}$ and $\mathrm{CD} 27^{\text {hi }} \mathrm{B}$ cells. $\mathrm{CD} 19^{+} \mathrm{CD} 27^{+} \mathrm{IgD}^{+} \mathrm{USM} \mathrm{B}$ cells carry features of postGC B cells, such as SHM in their B cell receptor, and can reenter the GC or rapidly differentiate into antibody-producing plasma cells (39), findings which are supported by our finding of IgG-expressing and/or $\mathrm{IgD}^{-} \mathrm{B}$ cells in CSF or periphery that are clonally related to USM B cells. The $\mathrm{CD} 27^{+} \mathrm{IgD}^{+} \mathrm{B}$ cell population may resemble splenic marginal zone B cells or B1 cells and provide a primary response against encapsulated bacteria (40) by generating diverse low-affinity IgM. Additional work will be necessary to understand the involvement of IgD-expressing memory B cell subsets in MS.

We also studied CXCL13 and CXCR5 in a small subcohort of patients and found detectable levels of CXCL13 only in CSF of patients with $\mathrm{Gd}^{+}$lesions, which is consistent with prior reports of increased CXCL13 during MS relapses (41). Thus, a B cell-attracting CSF environment appears to be generated during phases of disease activity, and an inverse CXCL13 gradient (i.e., higher in plasma, absent in CSF), as observed in $\mathrm{Gd}^{-}$patients, might promote $\mathrm{B}$ cell egress from the CNS during periods of remission. CSF CXCL13 may derive from actively demyelinating lesions (42) or from meningeal ectopic lymphoid sites (43). Only a minority of CD27 hi B cells in PB and CSF expressed CXCR5, the receptor for CXCL13, while the majority of other CSF and PB B cell subsets were CXCR5 ${ }^{+}$. Thus, the CXCL13/CXCR5 interaction may be generally involved in $\mathrm{B}$ cell recruitment to the CNS; however, it is specifically not involved in the recruitment of CD27hi B cells. Accordingly, CSF PCs more likely matured within the CNS/CSF compartment from plasma cell progenitors that were either present in this compartment or had newly entered the CNS during the phase of disease activity. We found the highest levels of CXCR5 on naive and USM B cells. Interestingly, we found USM and naive B cells in CSF with clonally related counterparts in the periphery and, particularly in the case of naive B cells, a striking absence of intra-CSF clonal relatedness to post-GC B cells. This observation could reflect nonspecific bystander recruitment of CXCR5-expressing B cells along a CXCL13 gradient and supports the presence of homeostatically proliferated naive B cells as previously described (44).

In summary, our data in treatment-naive MS patients suggest substantial influx and activation of diverse B cell populations during episodes of MS disease activity that may feed into ectopic lymphoid compartments, as described in patients with progressive MS $(16,18)$. The presence of OCB and CSF B cell repertoires, which likely existed prior to the disease activity period observed in our study, suggests long-term survival of B cells in the CNS compartment, which may facilitate peripherally triggered relapsing activity from within. Following completion of phase III trials $(5,6)$, anti-CD20 therapy has now been approved for the treatment of relapsing and progressive MS in the US. However, important questions remain regarding CD20-targeted B cell-depleting therapy: when to start treatment to not only limit disease activity but to most effectively delay or even prevent secondary progression? Should antiCD20 therapy be used as second-line therapy in patients with ongoing disease activity under another disease-modifying therapy, or should it be used as first-line therapy? Once established in the intrathecal (i.e., leptomeningeal or CNS parenchymal), compartment B cells may be less effectively targeted by peripher- 
A

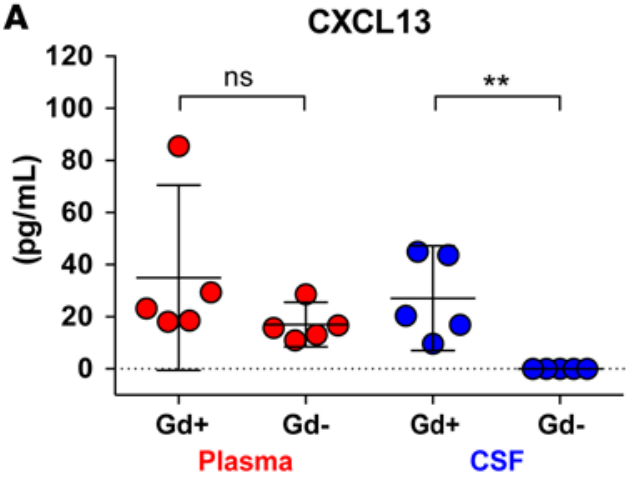

B

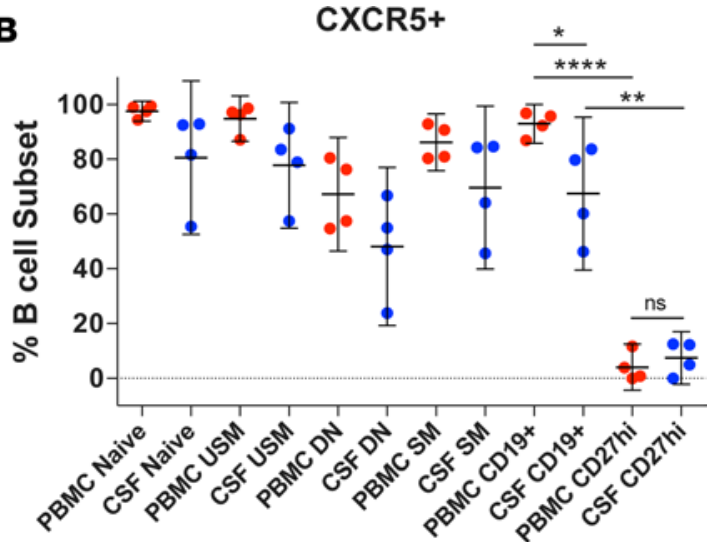

C

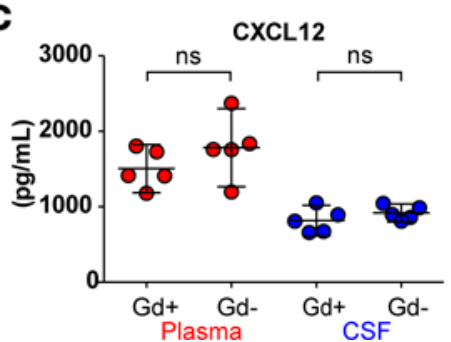

D

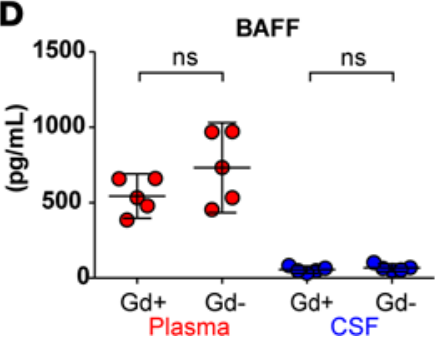

E

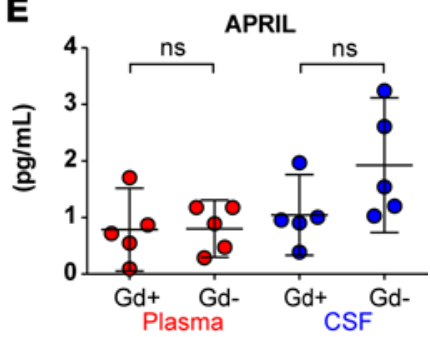

Figure 7. Locally produced CXCL13 may drive B cell recruitment to the CNS. CXCL13 was undetectable in CSF from Gd- patients, while CXCL13 levels in plasma and $\mathrm{Cd}^{+}$CSF were similar (A). CXCR5 is expressed on the majority of CSF and PBMC B cells (B). In combination, CXCR5 is present on more PBMCs than CSF CD19+ $\mathrm{B}$ cells and only expressed on a minority of PBMCs and CSF CD27 hi plasmablasts/plasma cells. There was no significant difference between CXCL12 (C), BAFF (D), and APRIL (E) in CSF from Gd ${ }^{+}$or $\mathrm{Gd}^{-}$patients. Overall CXCL12 and BAFF levels were higher in plasma than in CSF. Cytokine/chemokine concentrations were determined in CSF and plasma samples from $\mathrm{Gd}^{+}$and $\mathrm{Gd}^{-}$patients by ELISA ( $n=5$ per group; see Table 1$)$. Data are shown as scatter plots with mean $\pm 95 \% \mathrm{Cl}$. Comparisons were made using unpaired or paired $t$ tests; ${ }^{*} P<0.05,{ }^{* *} P<0.01,{ }^{* * *} P<0.0001$. N, naive B cells; USM, unswitched-memory B cells; DN, double-negative B cells; SM, switched-memory B cells; PC, plasma cells/plasmablasts.

ally acting B cell-depleting therapies (45). While anti-CD20 antibodies appear to be moderately effective in progressive MS $(6,46)$, onset of secondary progression may not be prevented if treatment is started too late (47). Therefore, it may be desirable to limit B cell influx into the CNS as early and effectively as possible to most successfully limit immune mechanisms that support MS progression.

\section{Methods}

Patients and samples. CSF and PB were sampled from a total of 39 patients recruited at the UCSF MS Center. All patients were treatment naive (i.e., no glucocorticoids or disease-modifying MS therapy at any time prior to lumbar puncture [LP] and MRI), sought medical care for a possible new diagnosis of MS, and met clinical and radiographic diagnostic criteria for RRMS or CIS. PB was obtained by venipuncture. CSF was obtained by standard LP; the first 1-3 $\mathrm{ml} \mathrm{CSF}$ were discarded and then CSF was obtained for clinical purposes. On average, $11.2 \mathrm{ml}( \pm 3.35 \mathrm{ml}, \mathrm{SD})$ of CSF were obtained for research studies at the end of the LP procedure. MRI imaging of the brain and spinal cord with and without $\mathrm{Gd}$ was obtained for each patient within \pm 1 day of their $\mathrm{LP}$; lesion counts ( $\mathrm{T} 1 \mathrm{Gd}^{+}$or $\mathrm{T} 2$ ) or volumes were not determined for this study.

Flow cytometry analysis and sorting. Peripheral mononuclear blood cells (PBMCs) were isolated using a Ficoll gradient, red blood cell lysis, and washing with phosphate-buffered saline (PBS) and $1 \%$ bovine serum albumin. PBMCs $\left(5 \times 10^{7}\right)$ and CSF cell pellets were blocked with FcR Block (Miltenyi Biotec) and incubated in the dark on ice for 20 minutes with the following antibodies: for PBMCs, we used IgD Brilliant Violet 421 (Biolegend 11-26c.2a), CD19 FITC (Biolegend HIB19), CD5 PerCP-eFluor710 (eBioscience YKIX322.3), CD38 PE (eBioscience 90), and CD27 APC (eBioscience O323) and for CSF, we used IgD Brilliant Violet 421 (Biolegend 11-26c.2a), CD20 FITC (Beckman Coulter B9E9), CD19 PC5.5 (Beckman Coulter J3-119), and CD3 PE (Beckman Coulter UCHT1). For the CXCR5-specific panels (PBMC and CSF), we used IgD Brilliant Violet 421 (Biolegend 11-26c.2a), CXCR5 FITC (eBioscience MU5UBEE), CD38 PerCPCy5.5 (BD Biosciences HIT2), CD3 PC7 (Beckman Coulter UCHT1), CD138 PE (Miltenyi Biotec B-B4), CD27 APC (eBioscience O323), and CD19 APC-Alexa Fluor 750 (Beckman Coulter J3-119). Cells were washed and resuspended in PBS con- 


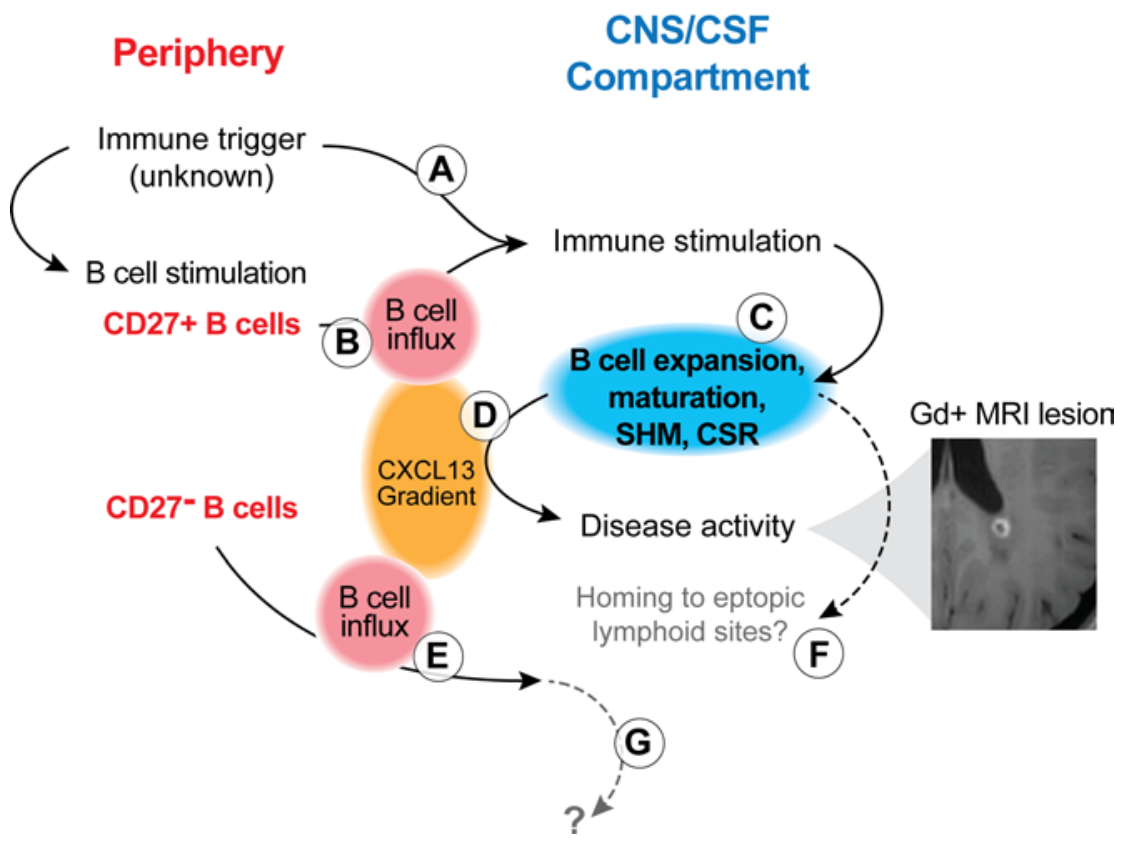

Figure 8. Model of B cell activation and involvement during MS disease activity. Peripheral immune activation can lead to intrathecal immune stimulation, either via antigen nonspecific (A; e.g., via cytokines during an infectious disease) or antigen-specific (B; via preformed CD27 $7^{+}$IgD- SM) mechanisms. This immune activation involves B and T cells, as supported by our flow cytometry findings and those of others (24) (Figures 1-3), and leads to B cell activation, modification of the B cell receptor (somatic hypermutation [SHM]; Ig-class switch recombination [CSR]) and B cell maturation (C). B cell influx and intrathecal B cell expansion are supported by Ig-RepSeq (Figures 5 and 6). These immune mechanisms support MS disease activity (evidenced by Gd ${ }^{+}$MRI lesions; example from patient 57514) and lead to increased production of CXCL13 (D), which, in turn, also attracts CD27- B cells to the CNS/CSF compartment (E). These CD27- B cells may not have been involved in the initial peripheral immune triggering event; rather, their migration to the CNS could be a nonspecific event. Activated CD27 $\mathrm{B}$ cells, once in the CNS, may further mature to antibody-secreting plasmablasts/plasma cells and may home to ectopic lymphoid sites (F). We found no evidence for $C D 27^{-} \mid g D^{+} B$ cells maturing to antigen-specific $C D 27^{+} B$ cell subset; the fate of naive B cells in $C S F$ remains unknown (G). It is important to note that several steps described in this model remain hypothetical and subject of future research.

taining $1 \%$ fetal bovine serum (Thermo Fisher Scientific), 1 mM EDTA, and $25 \mathrm{mM}$ HEPES and collected on a MoFlo Astrios (Beckman Coulter). B cell subsets were gated out of $\mathrm{CD} 19^{+}$and defined as naive $\mathrm{IgD}^{+} \mathrm{CD} 27^{-}$, USM IgD ${ }^{+} \mathrm{CD} 27^{+}, \mathrm{DN} \mathrm{IgD}^{-} \mathrm{CD} 27^{-}$, or $\mathrm{SM} \mathrm{IgD-CD} 27^{+}$. CD27 $7^{\text {hi }} \mathrm{PCs}\left(\mathrm{CD} 27^{\text {hi }}\right)$ were sorted out of the $\mathrm{PB}$ as IgD-CD27 $7^{\text {hi }} \mathrm{CD} 38^{\text {hi }}$ and out of the $\mathrm{CSF}$ as IgD-CD27 ${ }^{\text {hi }} . \mathrm{B}$ cells were sorted directly into RLT Buffer (Qiagen) with $1 \% \beta$-mercaptoethanol for cell lysis and RNA preservation and stored at $-80^{\circ} \mathrm{C}$.

PB B cell subsets (naive, USM, DN, SM, CD27 hi) were analyzed from all patients in this study and sorted and subjected to Ig-RepSeq from 11 patients (Table 1). CSF lymphocytes from all patients were analyzed by flow cytometry and either bulk sorted ( $n=3: 51113 \mathrm{a}, 51314 \mathrm{a}$, and $51814 \mathrm{a})$ or when possible sorted as 4 (naive, USM, DN, SM/CD27 combined: $n=2,56414 \mathrm{a}$ and 56514a) or 5 separate subsets ( $n=6: 56914 \mathrm{a}$, 57114a, 57414a, 57514a, 57714a, and 64215a).

Ig-RepSeq. Total RNA was extracted from sorted B cell subsets (RNeasy Micro Kit, Qiagen) and reverse transcribed (iScript cDNA Synthesis Kit, Bio-Rad). PB and CSF cDNA was used for IgM-VH and IgG-VH amplification by PCR (Advantage 2 PCR Kit, Clontech) using a pool of custom-designed IGHV1-7 family forward primers and IgG- or IgM-specific reverse primers with IonXpress barcodes to uniquely tag each subset/isotype prior to library prep (Table 2). PB samples were subjected to 30-35 cycles of PCR, and CSF subsets were amplified 35-50 cycles in increments of 5 cycles until product was detected by agarose gel. PCR-amplified product was purified using a 1.5\% agarose gel with Sybr Safe and extracted (MiniElute Kit, Qiagen). Gel-purified DNA was quantified (High Sensitivity DNA Kit and Bioanalyzer, Agilent Biotechnologies) and then diluted to $13.5-16 \mathrm{pM}$ to create an equimolar DNA library. The DNA library underwent emulsion PCR (Ion OneTouch2 Kit, Life Technologies) to bind and clonally expand DNA fragments onto IonSphere beads (ISP) for sequencing. Enriched ISPs were quality control checked using a Qubit Fluorometer and sequenced on the Ion PGM System (Life Technologies) using 318 v2 chips. Sequence files are available in fastq format at NCBI (BioProject accession PRJNA397295)

CSF and plasma cytokine/chemokine ELISAs. Concentrations of CXCL13, CXCL12, and BAFF were 
measured in plasma and CSF using commercially available ELISA kits from R\&D Systems, and concentrations of APRIL were determined using ELISA kits from eBioscience. All dilutions and sample measurements were performed according to the manufacturer's instructions. Optical density of each plate was read at $450 \mathrm{~nm}$ within 30 minutes using a microplate reader. The quantitative results were then statistically evaluated using a nonparametric $t$ test (Graphpad Prism).

MRI acquisition and analysis. MRI scans were acquired on a 3T Siemens scanner following a standardized protocol that included 3D T1-weighted magnetization-prepared Gradient echo images (T1-MPRAGE), 3D fluid-attenuated inversion recovery (FLAIR), and T2/PD images of the brain and T2-weighted and short tau inversion recovery (STIR) images of the spinal cord. Brain MPRAGE and T1-weighted spinal cord images were acquired after administration of gadopentetate dimeglumine. Readings were performed blinded to the clinical and immunological data.

Statistics and immune repertoire analysis. Statistical analyses were performed using GraphPad Prism. A 2-tailed $t$ test was used for linear measures and Fisher's exact test for group comparisons; $\mathrm{P}$ values of less than 0.05 were considered significant. $P$ values of less than 0.05 were considered statistically significant. Fastq sequence files were generated based on raw sequencing output files (BAM format) retrieved from the IonTorrent PGM Sequencer. For all reads, IGHV and IGHJ usage, H-CDR3 amino acid sequence, and numbers of SHM along the IGHV portion of each Ig-VH sequence were determined using a custom pipeline incorporating sequence alignments and MiXCR (48) (Note that the compiled bioinformatics pipeline is available for download; https://github.com/swuecho/Immune-repertoire-network). Clonally related IgM/G-VH sequences were clustered based on H-CDR3 similarity (Hamming distance $=2$; ref. 49) and usage of the same IGHV and IGHJ germline segments. Simplified views displaying the overall number of clusters per B cell subset and numbers of clonal connections (i.e., relationships) between subsets were visualized in Cytoscape version 3.3.0 (50). The goal of our analysis was to obtain information regarding the presence of clonally related Ig-VH derived from different B cell subsets in CSF and/or PB.

Study approval. All investigations were IRB approved (UCSF Committee on Human Research), and all patients provided written informed consent. This study was conducted in accordance with the Declaration of Helsinki.

\section{Author contributions}

ELE, BAM, and HCVB conceived of the project and designed the experiments. ELE, BAM, EL, ALG, and SD conducted experiments. ELE, BM, HW, SW, MRW, and HCVB conducted data analyses. CJB, AA, NSP, MK, BACC, MRW, and SLH recruited patients, provided patient data, and assisted with manuscript writing. $\mathrm{AB}$ and $\mathrm{RGH}$ performed MRI analyses, provided MRI data, and assisted with manuscript writing. ELE and HCVB wrote the manuscript.

\section{Acknowledgments}

The authors are deeply grateful to their patients who have agreed to participate in this research study and all members of the UCSF MS Expression, Proteomics, Imaging, Clinical (EPIC) study team who supported this study. Our studies were supported by grants from the National MS Society (RG4868 to HCVB), the NIH/National Institute of Neurological Disorders and Stroke (K02NS072288, R01NS092835 to HCVB), and the Valhalla Foundation as well as by gifts from the Friends of the Multiple Sclerosis Research Group at UCSF. HCVB was also supported by an endowment from the Rachleff Family Foundation. AB was supported by the Gottfried and Julia Bangerter-Rhyner Foundation and the Freiwillige Akademische Gesellschaft (Basel, Switzerland).

Address correspondence to: Michael R. Wilson, Weill Institute for Neurosciences, Department of Neurology, UCSF, 675 Nelson Rising Lane, Campus Box 3206, San Francisco, California 94158, USA. Phone: 415.502.7429: Email: Michael.Wilson@ucsf.edu.

HCVB's present address is: F. Hoffmann-La Roche, Basel, Switzerland.

\footnotetext{
1. Bar-Or A, et al. Rituximab in relapsing-remitting multiple sclerosis: a 72-week, open-label, phase I trial. Ann Neurol. 2008;63(3):395-400.
} 
2. Hauser SL, et al. B-cell depletion with rituximab in relapsing-remitting multiple sclerosis. $N$ Engl J Med. 2008;358(7):676-688.

3. Kappos L, et al. Ocrelizumab in relapsing-remitting multiple sclerosis: a phase 2, randomised, placebo-controlled, multicentre trial. Lancet. 2011;378(9805):1779-1787.

4. Sorensen PS, et al. Safety and efficacy of ofatumumab in relapsing-remitting multiple sclerosis: a phase 2 study. Neurology. 2014;82(7):573-581.

5. Hauser SL, et al. Ocrelizumab versus interferon beta-1a in relapsing multiple sclerosis. N Engl J Med. 2017;376(3):221-234

6. Montalban X, et al. Ocrelizumab versus placebo in primary progressive multiple sclerosis. N Engl J Med. 2017;376(3):209-220.

7. Bankoti J, et al. In multiple sclerosis, oligoclonal bands connect to peripheral B-cell responses. Ann Neurol. 2014;75(2):266-276.

8. Palanichamy A, et al. Immunoglobulin class-switched B cells form an active immune axis between CNS and periphery in multiple sclerosis. Sci Transl Med. 2014;6(248):248ra106.

9. Stern JN, et al. B cells populating the multiple sclerosis brain mature in the draining cervical lymph nodes. Sci Transl Med. 2014;6(248):248ra107.

10. von Büdingen HC, et al. B cell exchange across the blood-brain barrier in multiple sclerosis. J Clin Invest. 2012;122(12):4533-4543

11. Kowarik MC, et al. The cerebrospinal fluid immunoglobulin transcriptome and proteome in neuromyelitis optica reveals central nervous system-specific B cell populations. J Neuroinflammation. 2015;12:19.

12. Cepok S, et al. Short-lived plasma blasts are the main B cell effector subset during the course of multiple sclerosis. Brain. 2005;128(Pt 7):1667-1676.

13. Winges KM, Gilden DH, Bennett JL, Yu X, Ritchie AM, Owens GP. Analysis of multiple sclerosis cerebrospinal fluid reveals a continuum of clonally related antibody-secreting cells that are predominantly plasma blasts. J Neuroimmunol. 2007;192(12):226-234.

14. Baranzini SE, Jeong MC, Butunoi C, Murray RS, Bernard CC, Oksenberg JR. B cell repertoire diversity and clonal expansion in multiple sclerosis brain lesions. I Immunol. 1999;163(9):5133-5144.

15. Cross AH, Stark JL, Lauber J, Ramsbottom MJ, Lyons JA. Rituximab reduces B cells and T cells in cerebrospinal fluid of multiple sclerosis patients. J Neuroimmunol. 2006;180(1-2):63-70.

16. Absinta M, et al. Gadolinium-based MRI characterization of leptomeningeal inflammation in multiple sclerosis. Neurology. 2015;85(1):18-28.

17. Serafini B, Rosicarelli B, Magliozzi R, Stigliano E, Aloisi F. Detection of ectopic B-cell follicles with germinal centers in the meninges of patients with secondary progressive multiple sclerosis. Brain Pathol. 2004;14(2):164-174.

18. Howell OW, et al. Meningeal inflammation is widespread and linked to cortical pathology in multiple sclerosis. Brain. 2011;134(Pt 9):2755-2771.

19. Lehmann-Horn K, Wang SZ, Sagan SA, Zamvil SS, von Büdingen HC. B cell repertoire expansion occurs in meningeal ectopic lymphoid tissue. JCI Insight. 2016;1(20):e87234.

20. Villar LM, et al. Immunological markers of optimal response to natalizumab in multiple sclerosis. Arch Neurol. 2012;69(2):191-197.

21. von Glehn F, et al. Disappearance of cerebrospinal fluid oligoclonal bands after natalizumab treatment of multiple sclerosis patients. Mult Scler. 2012;18(7):1038-1041.

22. Harrer A, et al. Cerebrospinal fluid parameters of B cell-related activity in patients with active disease during natalizumab therapy. Mult Scler. 2013;19(9):1209-1212.

23. Ciccarelli O, Toosy AT. Conversion from clinically isolated syndrome to multiple sclerosis: A large multicentre study. Mult Scler. 2015;21(8):967-968.

24. Sandberg-Wollheim M, Turesson I. Lymphocyte subpopulations in the cerebrospinal fluid and peripheral blood in patients with multiple sclerosis. Scand J Immunol. 1975;4(8):831-836.

25. Corcione A, et al. Recapitulation of B cell differentiation in the central nervous system of patients with multiple sclerosis. Proc Natl Acad Sci USA. 2004;101(30):11064-11069.

26. Loman NJ, et al. Performance comparison of benchtop high-throughput sequencing platforms. Nat Biotechnol. 2012;30(5):434-439.

27. Kuenz B, et al. Cerebrospinal fluid B cells correlate with early brain inflammation in multiple sclerosis. PLoS One. 2008;3(7):e2559.

28. Michelutti A, et al. B-cell subsets in the joint compartments of seropositive and seronegative rheumatoid arthritis (RA) and No-RA arthritides express memory markers and ZAP70 and characterize the aggregate pattern irrespectively of the autoantibody status. Mol Med. 2011;17(9-10):901-909.

29. Ligocki AJ, et al. Expansion of CD27high plasmablasts in transverse myelitis patients that utilize VH4 and JH6 genes and undergo extensive somatic hypermutation. Genes Immun. 2013;14(5):291-301.

30. Hohlfeld R, Dornmair K, Meinl E, Wekerle H. The search for the target antigens of multiple sclerosis, part 2: CD8+ T cells, B cells, and antibodies in the focus of reverse-translational research. Lancet Neurol. 2016;15(3):317-331.

31. Obermeier B, et al. Matching of oligoclonal immunoglobulin transcriptomes and proteomes of cerebrospinal fluid in multiple sclerosis. Nat Med. 2008;14(6):688-693.

32. von Büdingen HC, Gulati M, Kuenzle S, Fischer K, Rupprecht TA, Goebels N. Clonally expanded plasma cells in the cerebrospinal fluid of patients with central nervous system autoimmune demyelination produce "oligoclonal bands". J Neuroimmunol. 2010;218(1-2):134-139.

33. Correale J, Fiol M, Gilmore W. The risk of relapses in multiple sclerosis during systemic infections. Neurology. 2006;67(4):652-659.

34. Wucherpfennig KW, Strominger JL. Molecular mimicry in T cell-mediated autoimmunity: viral peptides activate human T cell clones specific for myelin basic protein. Cell. 1995;80(5):695-705.

35. Polman $\mathrm{CH}$, et al. A randomized, placebo-controlled trial of natalizumab for relapsing multiple sclerosis. $N$ Engl J Med. 2006;354(9):899-910.

36. Beltrán E, et al. Intrathecal somatic hypermutation of IgM in multiple sclerosis and neuroinflammation. Brain. 2014;137(Pt 10):2703-2714

37. Qin Y, Duquette P, Zhang Y, Talbot P, Poole R, Antel J. Clonal expansion and somatic hypermutation of V(H) genes of B cells from cerebrospinal fluid in multiple sclerosis. J Clin Invest. 1998;102(5):1045-1050.

38. Ritchie AM, et al. Comparative analysis of the CD19+ and CD138+ cell antibody repertoires in the cerebrospinal fluid of 
patients with multiple sclerosis. J Immunol. 2004;173(1):649-656.

39. Seifert M, et al. Functional capacities of human IgM memory B cells in early inflammatory responses and secondary germinal center reactions. Proc Natl Acad Sci USA. 2015;112(6):E546-E555.

40. Weller S, et al. Human blood IgM "memory" B cells are circulating splenic marginal zone B cells harboring a prediversified immunoglobulin repertoire. Blood. 2004;104(12):3647-3654

41. Ragheb S, et al. Multiple sclerosis: BAFF and CXCL13 in cerebrospinal fluid. Mult Scler. 2011;17(7):819-829.

42. Krumbholz M, et al. Chemokines in multiple sclerosis: CXCL12 and CXCL13 up-regulation is differentially linked to CNS immune cell recruitment. Brain. 2006;129(Pt 1):200-211.

43. Magliozzi R, Columba-Cabezas S, Serafini B, Aloisi F. Intracerebral expression of CXCL13 and BAFF is accompanied by formation of lymphoid follicle-like structures in the meninges of mice with relapsing experimental autoimmune encephalomyelitis J Neuroimmunol. 2004;148(1-2):11-23.

44. Kinnunen T, et al. Specific peripheral B cell tolerance defects in patients with multiple sclerosis. J Clin Invest. 2013;123(6):2737-2741.

45. Komori M, et al. Insufficient disease inhibition by intrathecal rituximab in progressive multiple sclerosis. Ann Clin Transl Neurol. 2016;3(3):166-179.

46. Hawker K, et al. Rituximab in patients with primary progressive multiple sclerosis: results of a randomized double-blind placebo-controlled multicenter trial. Ann Neurol. 2009;66(4):460-471.

47. von Büdingen HC, et al. Onset of secondary progressive MS after long-term rituximab therapy - a case report. Ann Clin Transl Neurol. 2017;4(1):46-52.

48. Bolotin DA, et al. MiXCR: software for comprehensive adaptive immunity profiling. Nat Methods. 2015;12(5):380-381.

49. Hamming RW. Error detecting and error correcting codes. Bell Syst Tech J. 1950;29(2):147-160.

50. Shannon P, et al. Cytoscape: a software environment for integrated models of biomolecular interaction networks. Genome Res. $2003 ; 13(11): 2498-2504$. 